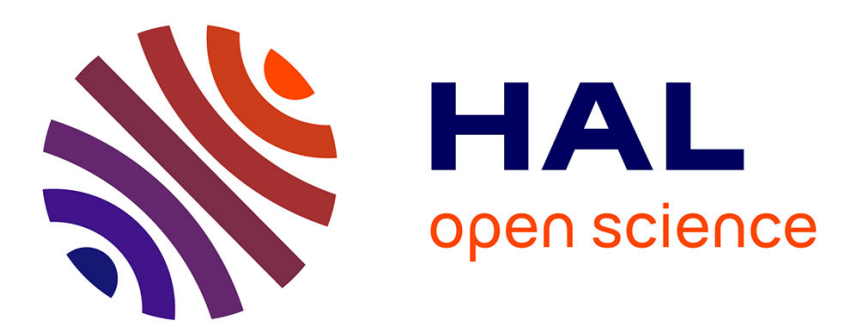

\title{
A Maxwell's demon in the generation of an intense and slow guided beam
}

G. Reinaudi, David Guéry-Odelin

\section{To cite this version:}

G. Reinaudi, David Guéry-Odelin. A Maxwell's demon in the generation of an intense and slow guided beam. Physical Review A : Atomic, molecular, and optical physics [1990-2015], 2008, 78, pp.015401. 10.1103/PhysRevA.78.015401. hal-00273918v2

\section{HAL Id: hal-00273918 https://hal.science/hal-00273918v2}

Submitted on 15 Sep 2008

HAL is a multi-disciplinary open access archive for the deposit and dissemination of scientific research documents, whether they are published or not. The documents may come from teaching and research institutions in France or abroad, or from public or private research centers.
L'archive ouverte pluridisciplinaire HAL, est destinée au dépôt et à la diffusion de documents scientifiques de niveau recherche, publiés ou non, émanant des établissements d'enseignement et de recherche français ou étrangers, des laboratoires publics ou privés. 


\title{
A Maxwell's demon in the generation of an intense and slow guided beam
}

\author{
G. Reinaudi ${ }^{1}$ and D. Guéry-Odelin ${ }^{1,2}$ \\ ${ }^{1}$ Laboratoire Kastler Brossel, Ecole Normale Supérieure, 24 rue Lhomond, 75005 Paris, France \\ ${ }^{2}$ Laboratoire Collisions Agrégats Réactivité, CNRS UMR 5589, IRSAMC, \\ Université Paul Sabatier, 118 Route de Narbonne, 31062 Toulouse CEDEX 4, France
}

(Dated: September 15, 2008)

\begin{abstract}
We analyze quantitatively the generation of a continuous beam of atoms by the periodic injection of individual packets in a guide, followed by their overlapping. We show that slowing the packets using a moving mirror before their overlapping enables an optimal gain on the phase space density of the generated beam. This is interpreted as a Maxwell's demon type strategy as the experimentalist exploits the information on the position and velocity of the center of mass of each packet.
\end{abstract}

PACS numbers: 32.80.Pj, 03.75.Pp

Over the last thirty years, there has been very significant and impressive progress in the experimental ability to increase the phase space density of atomic clouds, enabling the quantum degenerate regime to be reached [1].

All these advances can be revisited in terms of information entropy 2]. The powerful laser cooling technique [3] decreases dramatically the temperature and the entropy of an atomic cloud, at the expense of an increased disorder for the photons leaving the laser mode through spontaneous emission to populate other modes. The entropy of the global system made of \{atoms+photons increases as expected from the second law of thermodynamics [4]. In the evaporative cooling technique [5], the disorder of the system made of all particles involved from the beginning of the evaporation ramp increases each time a particle is evaporated, since this atom is no more localized in the trapping region. Accordingly, this technique yields a decrease of entropy for the subsystem made of the remaining trapped particles. Some of those techniques have also been implemented on atomic beams 6.

An optimized scheme for the implementation of informational cooling has been recently proposed in Ref. 7] and experimentally demonstrated in Ref. 8]: in this scheme, the increase of entropy of the radiation field in the scattering of a photon is exactly compensated by the reduction of entropy for the trapped atoms. Another strategy to increase the phase space density consists in changing adiabatically, and therefore isentropically, the density of states experienced by the atoms as demon-

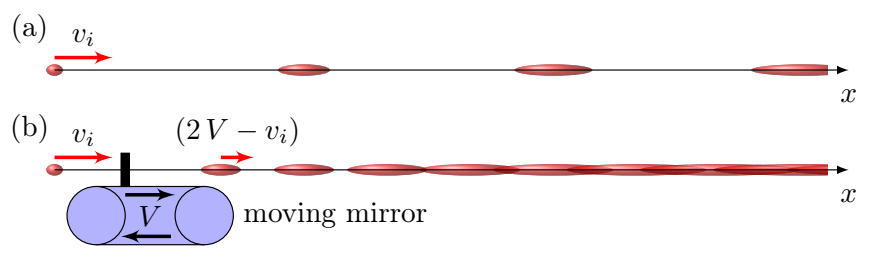

FIG. 1: (Color online) Schematic representation of the generation of a continuous beam by injecting packets into a guide (a), and by slowing them with a moving mirror (velocity $V$ ) before their overlapping (b). strated in [10]. The gain in information results from the transfer of population in the new set of low energy levels. The entropy $S$ is simply related to the phase space density $\rho$ by $S=-N k_{B} \log \rho+S_{0}$ [17]. If the shape of the confining potential is modified adiabatically, $S$ remains constant but $S_{0}$ changes which modifies in turn the phase space density $\rho[9,10]$.

Conversely, information can be used directly to increase the phase space density. This is realized in the stochastic cooling technique applied on a beam of charged particles in a storage ring [11]. Taking advantage of the particle's charge, information is extracted in one place and an adapted feedback action in another place is exerted later on. This technique seems at first sight to violate the Liouville theorem which states the incompressibility of phase volume when only conservative forces are involved. However particles being point like, there is a lot of empty space between them. Each particle can in principle be manipulated individually to increase the phase space density. This requires all information about position and velocity of the particles. Such a procedure resembles Maxwell's demon thought experiment [12]. There is no violation of the second law of thermodynamics since the measurement performed by the demon implies an entropy increase [13].

It is definitely more difficult to extract information on a beam made of neutral particles. We show in this article that a recently published optimization [14] of the technique presented in Ref. [16] to generate a continuous beam by periodically injecting packets of atoms in a guide is reminiscent of Maxwell's demon strategy. The generation of an intense and slow guided beam involves two conflicting requirements: the high flux implies coupling packets at a high repetition rate, and the low velocity requirement limits this rate. An upward potential hill can be used to slow down the beam [6]. However, a better strategy from the point of view of the phase space density of the generated beam consists in slowing down the packets by letting them undergo an elastic collision with a moving potential barrier before their overlapping 14, 15] (see Fig. 1). The reason why this latter scheme can be better than the former one in terms of entropy is that it corresponds to the realization of a true Maxwell's 
demon with the use of information on the center of mass of the packet before the overlapping.

For the sake of simplicity and without loss of generality, the argument is presented quantitatively for a onedimensional system. However, all results derived in this paper are valid when one takes into account explicitly the transverse degrees of freedom, as long as the transverse confinement is not modified or only modified adiabatically by the presence of the mirror, and the initial velocity dispersion is the same on all degrees of freedom.

We assume the successive packets to be identical and uniformly distributed over a rectangular surface in the one-dimensional phase-space. The packets are then characterized by four parameters: the number of atoms $N$, the initial size $\Delta x$, the velocity dispersion $\Delta v$ and the center of mass velocity $v_{i}$. The phase space density of a given packet reads:

$$
\rho_{p}=\frac{N \hbar}{m \Delta x \Delta v}
$$

where $m$ denotes the mass of the atoms. Figure 2(a) represents a plot of $\rho_{p}$ in the one-particle phase space at different times. As expected from the Liouville theorem, the surface occupied by the packet remains constant. However its size in position space increases with time as a result of the velocity dispersion, or in other terms, velocity-position correlations are produced in the course of the time evolution of the packets. We consider such packets launched periodically with a constant time separation $\tau$. After a sufficient duration, they overlap, thermalize and form a continuous beam [16]. This beam in thermodynamic equilibrium is characterized by an atomic density $N /\left(v_{i} \tau\right)$ and the same velocity dispersion $\Delta v$ as the one of the packets [18]. The phase space

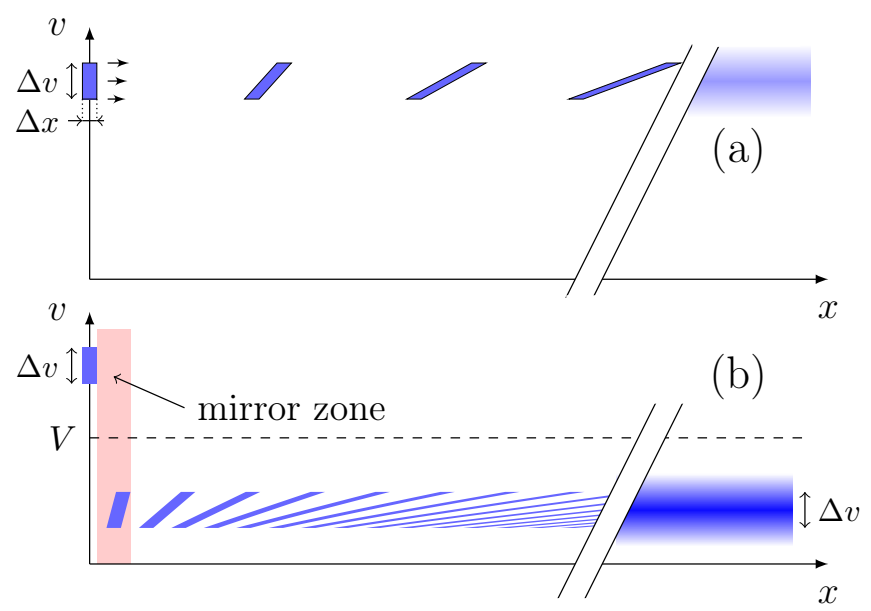

FIG. 2: (Color online) Representation in the single-particle phase space of one-dimensional atomic packets periodically injected in a guide. a) in the absence of slowing b) in the presence of slowing with the mirror moving at a velocity $V$ (dashed line). For a sufficiently long propagation distance, packets have merged and the thermal equilibrium for the corresponding beam is reached. density of the thermalized beam is given by:

$$
\rho=\frac{N \hbar}{m v_{i} \tau \Delta v} .
$$

The factor term $N / v_{i} \tau$ corresponds to the mean atomic density of the beam. From Fig. 2(a), we immediately conclude that $\rho<\rho_{p}$. This inequality reflects the Liouville theorem. It can be interpreted physically using the concept of information entropy [2]. Before overlapping, the packets are distinguishable $\left(v_{i} \tau>\Delta x\right)$, therefore the center of mass of each packet is well-defined. The overlapping accompanied by elastic collisions between successive packets corresponds to a loss of information on the center of mass of the packets. This merging process yields an increase of entropy or equivalently a decrease of the mean phase space density of the beam generated from the packets compared to the one of each packet.

An important feature of Eq. (2) is that the mean velocity of the packet enters explicitly the expression of the phase space density of the beam. In Ref. [14], this velocity dependence was exploited to realize a slow and intense guided atomic beam. Each atomic packet was slowed down by means of a moving mirror well-synchronized with the motion of the atomic packet. The overlapping occurred after this manipulation of each packet. This experimental trick permits one to ensure a high flux while having, in the end, a very low mean velocity for the beam generated from the slowed packets.

Such a specific action on each packet is reminiscent of the Maxwell's demon thought experiment. In Maxwell's scheme, the apparent violation of the second law of thermodynamics is made by exploiting information about particle's velocity. The experimentalist acts as a Maxwell's demon by exploiting all the information (position and velocity) on the center of mass of each packet to synchronize the motion of a moving mirror with which they will undergo an elastic collision 19]. The macroscopic mirror absorbs the microscopic momentum kick due to the reflection of the packet in the mirror's frame.

The reflection of a succession of slowed packets is represented on Fig. 2(b). In the single-particle phase space, it corresponds essentially to a translation while keeping its volume constant. The phase space density of the beam generated from those slowed packets is given by

$$
\rho^{\prime}=\frac{N \hbar}{m\left(2 V-v_{i}\right) \tau \Delta v}=\rho \frac{v_{i}}{\left(2 V-v_{i}\right)}>\rho,
$$

where $\left(2 V-v_{i}\right)$ represents the mean velocity of the beam made of the packets that have been slowed down through their interaction with the moving mirror. For the same repeating rate $\tau^{-1}$, a significant gain on the phase space density of the generated beam is therefore achievable.

The upper bound on phase space density of the beam generated from packets is given by the phase space density of each packet: $\rho^{\prime}<\rho_{p}$. This holds even if one uses time dependent potentials to manipulate them before their overlapping. In this context, there is no possible strategy to overcome this limit 2], since we do not 
use for each packet information at a microscopic scale i. e. on atoms individually.

For a given set of packet parameters $\left(N, v_{i}, \Delta v, \Delta x\right)$, one may wonder what is the optimum choice of mirror velocity $V$ to maximize the phase space density $\rho^{\prime}$ of the beam generated from the slowed packets, and what is the expression for this optimum depending on the experimental parameters.

To answer those questions, we will consider a specific example which contains all the relevant physical ingredients. As mentioned above, we model the initial packet by a uniform phase space density with an initial rectangular shape. The coordinates of the four vertices are $\left(\Delta x / 2, v_{i}+\Delta v / 2\right),\left(-\Delta x / 2, v_{i}+\Delta v / 2\right),\left(\Delta x / 2, v_{i}-\Delta v / 2\right)$ and $\left(-\Delta x / 2, v_{i}-\Delta v / 2\right)$. The mirror velocity $V$ has two constraints: the lowest velocity of the initial packet needs to be larger than the mirror velocity $v_{i}-\Delta v / 2>V$, and the lowest final velocity after interaction with the mirror should be positive to ensure the propagation of the beam in a well-defined direction $2 V-v_{i}-\Delta v / 2>0$. Let us introduce the dimensionless parameters $y=\Delta v / v_{i}$ and $z=V / v_{i}$ that are related respectively to the packet and the mirror. The two previous conditions on the mirror velocity are recast in the form:

$$
\frac{1}{2}+\frac{y}{4} \leq z \leq 1-\frac{y}{2}
$$

To maximize the flux of the continuous beam resulting from the overlapping of the periodically injected atomic packets, one needs to maximally decrease the time period $\tau$ between two successive packets [14]. However, $\tau$ cannot be chosen arbitrary small since the mirror is moving faster than the slowed packets and should not push and thus accelerate some of them while interacting with the next packet. In the following, we denote $\tau_{\min }$ the minimum repeating time that enables the mirror to slow down a given packet while not affecting the preceding slowed packet. This quantity depends on $\left(\Delta x / v_{i}\right), y$ and $z$. To work out its explicit expression, we model the mirror by an infinitely high and thin potential barrier. The mirror is periodically moving at a velocity $V$ over a distance allowing the slowing of all atoms of each packet and we assume that it acts on a given packet as soon as it is released (see Fig. 2b). We find:

$$
\tau_{\min }(y, z)=\frac{\Delta x}{v_{i}} \frac{(z-1-y / 2)}{(2 z-1-y / 2)(z-1+y / 2)}
$$

Using this result with Eqs. (1) and (3), we infer the maximum increase in phase space density:

$$
R_{\max }(y, z)=\frac{\rho_{\max }^{\prime}}{\rho_{p}}=\frac{\Delta x}{\left(2 V-v_{i}\right) \tau_{\min }(y, z)} .
$$

For given experiment, the dimensionless parameter $y$ is fixed. The optimum value of the mirror velocity $V^{*}$ is obtained by maximizing $R_{\max }(y, z)$ as a function of $z$. Taking into account the constraints (4), the equation $\partial R_{\max }(y, z) / \partial z=0$ gives a unique solution $z^{*}(y)$ :

$$
V^{*}=v_{i} z^{*}(y)=\frac{v_{i}}{6}\left(2+y+\sqrt{2} \sqrt{2-y-y^{2}}\right),
$$

and the domain in $y$ for which a solution exists is: $y<$ $2 / 3$. This condition simply means that the initial mean velocity has to be large enough compared to the velocity dispersion, as intuitively expected.

As illustrated on Fig. 3(a) by plotting $z^{*}(y)$ from Eq. (17), a remarkable feature of the optimal velocity for the mirror is that it is nearly constant over its validity domain and approximately equal to $2 v_{i} / 3$. We conclude from Eq. 3 that an optimal use of the mirror technique permits to gain a factor on the order of three on the phase space density generated from the packets compared to the value obtained in the same conditions but in the absence of the mirror.

Figure 3(b) shows that when $\Delta v / v_{i}$ tends to zero, the phase space density of the continuous flow tends to its upper bound, i.e. the phase space density of the individual packets. This corresponds to a situation where the slowed packets cover the single atom phase space in a quasi compact manner.

In practice, two effects tends to reduce the gain on the phase space density of a beam generated from slowed packet compared to the one without slowing [14]: (i) the finite thickness of the mirror, and (ii) a free propagation of the packets before their interaction with the mirror. We evaluate separately their effect in the following.

We denote $\Delta_{\mathrm{m}}$ the thickness of the mirror. For example, $\Delta_{\mathrm{m}} \sim 10 \mathrm{~cm}$ in the experiment described in Ref. 14]. The calculations performed previously can be readily adapted to take into account the size of the mir-

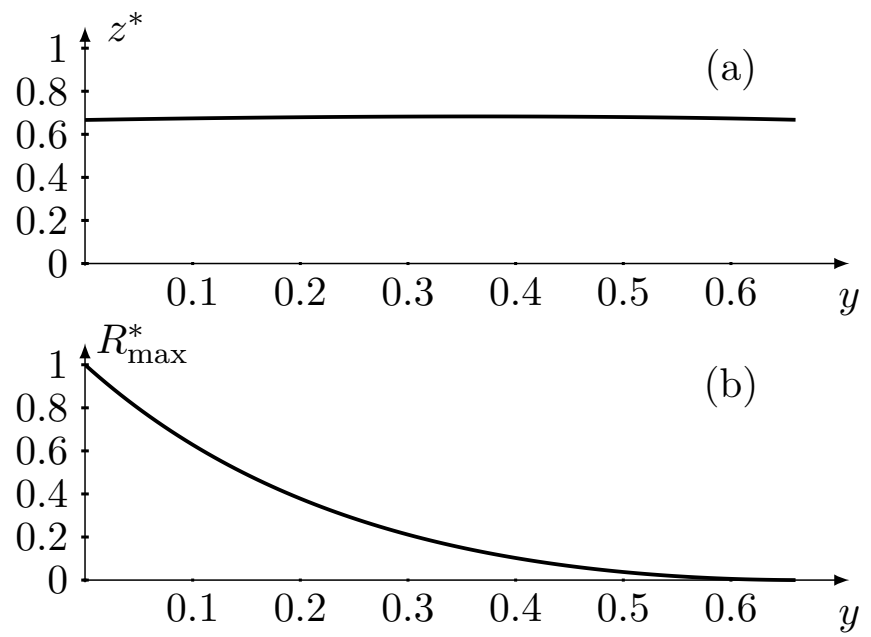

FIG. 3: (a) The optimal mirror velocity $z^{*}(y)=V^{*} / v_{i}$ normalized to $v_{i}$ is plotted as a function of the dimensionless parameter $y=\Delta v / v_{i}$ and (b) the corresponding maximum phase space density $R_{\max }^{*}=R_{\max }\left(y, z^{*}(y)\right)=\rho^{\prime} / \rho_{p}$ of a beam generated from the packet after their optimal slowing down and normalized to the packet initial phase space density, is plotted as a function of $y$. 
ror. In the limit $y \rightarrow 0$, the maximum gain on phase space density $R_{\max }^{\prime}\left(y, z^{*}(y)\right)$ saturates to $1 /\left(1+\Delta_{\mathrm{m}} / \Delta x\right)$. Indeed, the incompressible distance $\Delta_{\mathrm{m}}$ dictates an upper limit on the achievable atomic density. In addition, the optimal mirror velocity $V^{*}$ tends to $v_{i}$ as $\Delta_{\mathrm{m}}$ increases, which reflects the reduction of the gain on phase space density resulting from the limit on the atomic density.

Another experimental parameter to be considered lies in the fact that the atomic cloud cannot usually be slowed down just after its injection, but has to propagates freely over a distance $D$ before interacting with the mirror 20 ]. For example, one has $D \sim 25 \mathrm{~cm}$ in Ref. 14]. In this instance and assuming that $\Delta_{\mathrm{m}}=0$ for sake of simplicity, the general expression for the maximum of the ratio $\rho^{\prime} / \rho_{p}$ takes the form:

$$
R_{\max }^{\prime}(y, z, D)=\frac{R_{\max }(y, z)}{1+y \frac{D}{\Delta x}} .
$$

This result just reflects the fact that the packets have spread during their free propagation before interacting with the mirror, which reduces as expected the gain compared to the one without free flight. From Eq. (8), we conclude that the optimal velocity $V^{*}$ is the same as the one calculated Eq. (7).

In conclusion, we have investigated quantitatively an optimal strategy to produce a continuous beam with individual packets, in order to maximize the phase space density of the beam. The use of a mirror to slow down the packets before their overlapping can be interpreted as a Maxwell's demon type strategy, where the mirror acts as an active valve that modifies the properties of the packet by reducing its mean velocity. This study exemplifies, in the context of an atomic beam made of neutral atoms, the usefulness of the link between information, entropy and phase-space densities for designing optimal strategies.

We are indebted to J. Dalibard, T. Lahaye, M. Jeppesen, R. Mathevet, G. L. Gattobigio and A. Ridinger for useful comments. We acknowledge financial support from the Délégation Générale pour l'Armement (contract number 05-251487), the Institut Francilien de Recherche sur les Atomes Froids (IFRAF) and the Plan-Pluri Formation (PPF). G. R. acknowledges support from the Délégation Générale pour l'Armement.
[1] E. A. Cornell and C. E. Wieman, Rev. Mod. Phys. 74, 875 (2002); W. Ketterle, Rev. Mod. Phys. 74, 1131 (2002).

[2] W. Ketterle and D. E. Pritchard, Phys. Rev. A 46, 4051(1992).

[3] S. Chu, Rev. Mod. Phys. 70, 685 (1998); W. D. Phillips, Rev. Mod. Phys. 70, 721 (1998); C. N. Cohen-Tannoudji, Rev. Mod. Phys. 70, 707 (1998).

[4] S. J. van Enk and G. Nienhuis, Phys. Rev. A 46, 1438 (1992).

[5] H. F. Hess, Phys. Rev. B 34, 3476 (1986); N. Masuhara, J. M. Doyle, J. C. Sandberg, D. Kleppner, T. J. Greytak, H. F. Hess, and G. P. Kochanski, Phys. Rev. Lett. 61, 935 (1988); W. Petrich, M. H. Anderson, J. R. Ensher, and E. A. Cornell, Phys. Rev. Lett. 74, 3352 (1995).

[6] T. Lahaye, Z. Wang, G. Reinaudi, S. P. Rath, J. Dalibard and D. Guéry-Odelin, Phys. Rev. A. 72, 033411 (2005).

[7] A. Ruschhaupt, J. C. Muga and M. G. Raizen, J. Phys. B: At. Mol. Opt. Phys. 39, 3833 (2006).

[8] G. N. Price, S. T. Bannerman, K. Viering, E. Narevicius, and M. G. Raizen, Phys. Rev. Lett. 100, 093004 (2008).

[9] P. W. Pinkse, A. Mosk, M. Weidemüller, M. W Reynolds, T. W. Hijmans, and J. T. Walraven, Phys. Rev. Lett. 78, 990 (1997).

[10] D. M. Stamper-Kurn, H.-J. Miesner, A. P. Chikkatur, S. Inouye, J. Stenger, and W. Ketterle, Phys. Rev. Lett. 81, 2194 (1998).

[11] S. van der Meer, Rev. Mod. Phys. 57, 689 (1985).

[12] J. C. Maxwell, Theory of Heat (Longmans, London, 1871), 4th edn, pp. 328-329.

[13] L. Szilard, Z. Phys. 53, 840 (1929).

[14] G. Reinaudi, Z. Wang, A. Couvert, T. Lahaye and D. Guéry-Odelin, Eur. Phys. J. D 40, 405 (2006).

[15] E. Narevicius, A. Libson, M. F. Riedel, C. G. Parthey,
I. Chavez, U. Even, and M. G. Raizen, Phys. Rev. Lett. 98, 103201 (2007).

[16] T. Lahaye, J. M. Vogels, K. Guenter, Z. Wang, J. Dalibard, and D. Guéry-Odelin, Phys. Rev. Lett. 93, 093003 (2004).

[17] According to the canonical formalism in statistical physics, the one particle phase space density is $\rho=N / z$, where $z=h^{-3} \int d^{3} r d^{3} p \exp \left(-\mathcal{H}(\mathbf{r}, \mathbf{p}) / k_{B} T\right)$ and $\mathcal{H}(\mathbf{r}, \mathbf{p})$ is the one particle hamiltonian. The entropy $S$ is obtained through the expression for the free energy $F=U-T S$, from which we infer the value of the term $S_{0} /\left(N k_{B}\right)=$ $1+U /\left(N k_{B} T\right)$. If the shape of the trapping potential changes, the mean energy $U$ and therefore $S_{0}$ change also.

[18] Strictly speaking, the thermalization cannot occur in a one-dimensional system. We assume that thermalization occurs which requires the problem to be three (or two) dimensional. The effective one dimensional treatment carried out in this article is valid if the longitudinal velocity dispersion is unchanged after thermalization. It is the case if the velocity dispersion along all degrees of freedom is initially the same

[19] The system made of the succession of the packets can be regarded as the one of a succession of macro-particles, each macro-particle being a packet. The action of the Maxwell's demon in this context consists in increasing the linear density of the macro-particles by slowing them down one after another.

[20] Indeed, a finite time for the preparation of a packet is required and one should ensure that the preparation of a given packet does not affect the previous packets, for instance through scattered light from the magneto-optical trap used to prepare the packets [16]. 